\title{
Cultural Materialism and Behavior Analysis: An Introduction to Harris
}

\author{
Brian D. Kangas \\ University of Florida
}

The year 2007 marks the 80th anniversary of the birth of Marvin Harris (1927-2001). Although relations between Harris' cultural materialism and Skinner's radical behaviorism have been promulgated by several in the behavior-analytic community (e.g., Glenn, 1988; Malagodi \& Jackson, 1989; Vargas, 1985), Harris himself never published an exclusive and comprehensive work on the relations between the two epistemologies. However, on May 23rd, 1986, he gave an invited address on this topic at the 12th annual conference of the Association for Behavior Analysis in Milwaukee, Wisconsin, entitled Cultural Materialism and Behavior Analysis: Common Problems and Radical Solutions. What follows is the publication of a transcribed audio recording of the invited address that Harris gave to Sigrid Glenn shortly after the conference. The identity of the scribe is unknown, but it has been printed as it was written, with the addendum of embedded references where appropriate. It is offered both as what should prove to be a useful asset for the students of behavior who are interested in the study of cultural contingencies, practices, and epistemologies, and in commemoration of this 80th anniversary.

Key words: cultural materialism, radical behaviorism, behavior analysis

\section{Cultural Materialism and Behavior Analysis: Common Problems and Radical Solutions}

\author{
Marvin Harris \\ University of Florida
}

Cultural materialism is a research paradigm which shares many epistemological and theoretical principles with radical behaviorism. Like radical behaviorism, it stresses environment over heredity, in opposition to biological reductionist paradigms. And like radical behaviorism, it stands in opposition to psychological paradigms such as cognitivism, which stress mind as the cause of behavior. It is not clear, however, to what extent cultural materialism and radical behaviorism have a comparable position vis-à-vis the anthropological analogue of cognitivism, which I call cultural idealism, for reasons that will become clear later on.

In fact, it is my contention that while cultural materialism and radical behaviorism have a common basis

Reprints may be obtained from Brian D. Kangas, Department of Psychology, University of Florida, P.O. Box 112250, Gainesville, Florida 32611-2250 (e-mail: kangas@ufl.edu). in rejection of mind as a cause of individual human behavior, radical behaviorism is not radically behaviorist enough for a confrontation with idealists' anthropological paradigm, in spite of the fact that cultural idealists identify mind as the locus of sociocultural reality and causality. The cause of this disparity lies in the radical behaviorist's neglect of the systemic properties of cultural phenomena and a consequent failure to identify the selective core of the sociocultural systems and how that selection takes place.

In the history of the development of paradigmatic attempts to solve the question of sociocultural causality, cultural materialism has confronted and continues to confront a set of long-established, common-sense but erroneous and unproductive beliefs about human culture that are similar to those which confronted and continue to confront the development of 